\title{
L'CEuvre des ouvriers. Lettre de Pauline Jaricot (décembre 1848)
}

Daniel Murat

\section{(2) OpenEdition \\ 1 Journals}

Édition électronique

URL : https://journals.openedition.org/clio/96

DOI : $10.4000 /$ clio.96

ISSN : 1777-5299

Éditeur

Belin

Édition imprimée

Date de publication : 1 avril 2002

Pagination : 190-197

ISBN : 2-85816-620-X

ISSN : $1252-7017$

Référence électronique

Daniel Murat, "L'CEuvre des ouvriers. Lettre de Pauline Jaricot (décembre 1848) », Clio. Histoire, femmes et sociétés [En ligne], 15 | 2002, mis en ligne le 08 février 2005, consulté le 28 avril 2022. URL : http:// journals.openedition.org/clio/96; DOI : https://doi.org/10.4000/clio.96 


\section{L'Euvre des ouvriers. Lettre de Pauline Jaricot (décembre 1848)}

Daniel MURAT

Le 25 février 1963 Jean XXIII a déclaré Pauline Jaricot "Vénérable», ce qui signifie que l'Église catholique s'engage à la béatifier. Depuis la sanctification de cette laïque, le comportement de l'Église est assez ambigu : elle loue son action auprès du monde ouvrier durant la première moitié du XIX siècle, mais elle ne publie pas ses analyses sur les causes de la paupérisation ouvrière, ni ses propositions pour y remédier. Cette situation fait que l'historien, pour appréhender l'engagement social de Pauline Jaricot, doit avoir recours aux documents fournis par ses biographes officiels.

Le texte de cette lettre - qui n'est pas présentée ici dans son intégralité - est extrait de la première biographie de Pauline Jaricot rédigée par Julia Maurin et 
publiée en $1892^{1}$. Pour reconstituer le texte de cette lettre qui, dans l'ouvrage, est entrecoupé par le récit de l'auteure, j'ai utilisé les informations fournies par deux autres auteurs. Ainsi, David Lathoud, dans le deuxième tome d'un ouvrage déjà ancien (1936), a reproduit toute la première partie de la lettre ${ }^{2}$, en précisant que cette retranscription était conforme à l'original se trouvant dans le dossier du "Procès informatif en vue de la béatification". Quant au Père Jean Servel, il a, dans un livre paru en 1963, reproduit ces extraits en mentionnant la référence des sources manuscrites ${ }^{3}$. Cette lettre a été datée par David Lathoud. Il indique que sa destinataire, Julia Maurin, avait été envoyée par Pauline Jaricot pour chercher l'appui de l'évêque de la Rochelle, à la suite de la première faillite de l'usine de Rustrel, un établissement qui devait servir d'exemple pour l' CEuvre des ouvriers.

Pauline Jaricot est née à Lyon, le 22 juillet 1799, dans une famille de riches négociants. Son père, commis durant la Révolution, avait spéculé en achetant des tissus alors que les prix s'étaient effondrés et il avait profité de la reprise économique sous le Consulat pour bâtir sa fortune. La famille Jaricot, mal acceptée par la vieille bourgeoisie lyonnaise, était en même temps très liée aux milieux catholiques légitimistes et elle figurait parmi les familles les plus en vue à Lyon au début du XIX $X^{e}$ siècle. La jeunesse de Pauline est identique à celle de toutes les jeunes filles de son milieu, du moins jusqu'à la mort de sa mère en 1816, événement douloureux, véritable tournant dans son existence. Elle décide alors de consacrer sa vie aux pauvres, revêt l'habit des ouvrières en soierie et crée une communauté de laïques «les Filles de Marie». Même si cette communauté fonctionne comme une congrégation, Pauline n'en fera pas une communauté de religieuses. En 1818, avec son frère Philéas, elle organise "la collecte du sou de la mission» auprès des ouvrières de la filature de sa sœur à St-Vallier. En 1821, cette association compte près de 1000 donateurs et gère des sommes importantes. En 1822, des notables lyonnais de la Congrégation des Messieurs s'emparent de cette association qui est dénommée Congrégation pour la propagation de la foi et écartent la jeune femme. Répondant à l'appel de Léon XII, elle lance en 1825 l'Association du Rosaire Vivant qui a deux objectifs : "réciter sans interruption le

1 Julia Maurin, Vie nouvelle de Pauline Jaricot, 2 tomes, Paris, Editions Victor Palme, 1892, page 312 et suivantes. Julia Maurin a été l'une des dernières fidèles de Pauline Jaricot. Elle fut élevée chez les Ursulines de Chavagnes en Vendée et exerça la profession d'institutrice.

2 David Lathoud, Marie Pauline Jaricot, 2 tomes, Paris, Editions la Bonne Presse, 1936.

3 Jean Servel, Un autre visage - textes inédits de Pauline Jaricot, Lyon, Editions du Chalet, 1963. 
chapelet dans toute la France et permettre la diffusion d'ouvrages de piété ou de vie de Saints». En 1834 l'association regroupe en France 11 millions de membres, chacun versant une cotisation variable selon ses revenus d'où le fait que Pauline Jaricot va gérer des sommes importantes dans une certaine opacité. À partir de 1829 , elle envoie régulièrement des circulaires aux membres de l'association, dans lesquelles elle commente les écritures saintes mais elle y développe aussi ses analyses concernant la condition ouvrière en France. Deux ans plus tard, en 1831, elle essaie de sensibiliser les associés du Rosaire Vivant pour créer ce qu'elle nomme l'CEuvre des ouvriers qui doit, selon elle, se structurer autour de deux pôles : une banque de prêts dénommée Banque du ciel, qui offrirait des prêts sans intérêt aux ouvriers, mais aussi aux congrégations et des entreprises industrielles où les ouvriers seraient traités humainement et recevraient un salaire suffisant.

Sa dénonciation de la condition ouvrière n'est pas isolée au sein de l'Église Catholique. Nombreuses sont les voix qui s'élèvent dans les années 1840 pour dénoncer la misère ouvrière. En 1845, Mgr Bonald, archevêque de Lyon, dénonce dans ses homélies "le faste qui est une insulte à la misère ouvrière». Mgr Giraud, évêque de Montpellier, affirme en 1846 "le droit du travailleur à un salaire qui suffise à son entretien et à un excédent qui lui vienne en aide au temps de la maladie, de la vieillesse, de l'interruption de travail»". Mais cette prise de conscience du malheur ouvrier n'empêche pas l'Église - institution - de se rallier en 1848 au parti de l'Ordre. À la même époque, Pauline Jaricot, sans remettre en cause ces choix institutionnels, n'a pas hésité à investir sa fortune et les sommes du Rosaire dans son CEuvre des ouvriers, non sans susciter une certaine hostilité dans la bourgeoisie lyonnaise qui voit là un risque de déstabilisation des autres entreprises. Et puis, en tant que femme elle transgresse un ordre social pensé par les hommes, qu'ils soient d'Église ou non. Enfin, par ses écrits, elle s'inscrit dans un domaine dont les femmes étaient en règle générale exclues. Finalement, en 1847, elle investit dans une fonderie à Rustrel dans le Vaucluse pour donner vie à son CEuvre des ouvriers, mais l'année suivante, c'est la première liquidation de la société gérante. Pauline Jaricot s'obstine mais l'expérience se termine en 1852 par sa faillite personnelle et celle de petits porteurs qui ont investi dans cette aventure, une faillite qui la met au ban de la société lyonnaise. Cet échec libère en quelque sorte la parole de Pauline Jaricot qui va développer ses critiques d'un système engendrant la pauvreté ouvrière. En 1852, elle adresse à l'Archevêque de

4 Gérard Cholvy, Histoire religieuse de la France contemporaine, Toulouse, Privat, 1985, pp. 68-69. 
Lyon et au Pape un mémoire intitulé "Adresse à la Sainte Église» où elle développe son analyse concernant la paupérisation de la classe ouvrière et ce que devrait être une cuvre qui engagerait toute l'Église.

Le regard de Pauline Jaricot sur la situation de la "classe ouvrière» est sans concession pour le patronat mais aussi pour le pouvoir politique en place. L'ouvrier qu'elle décrit est un homme exploité, réduit en quelque sorte à l'état d'esclavage par les patrons des fabriques. Cette misère a d'abord pour cause le comportement des patrons qui ne respectent plus le message de l'Évangile porté par le Christ qui a "choisi la pauvreté et le travail, afin de guérir toutes convoitises de la nature déchuem. Cette critique des possédants va plus loin que les remises en cause des évêques. En effet, même si Pauline Jaricot rejoint le discours d'une partie de l'élite religieuse, dénonçant la misère croissante des ouvriers, elle fait valoir que la possession de l'or et de l'argent a à voir avec le diable - «l'or et l'argent dont le dragon de l'abîme fait sa litière». Mais cette dénonciation ne l'empêche pas d'approcher le monde ouvrier dans un souci d'évangélisation. Elle va même jusqu'à proposer que l'Église forme des prêtres missionnaires issus du monde ouvrier, idée qu'elle développe dans une autre lettre adressée à Julia Maurin en décembre 1849.

Malgré un vocabulaire aux connotations socialistes - "Capitalistes spéculateurs, les riches contentent leur besoin de jouissance», Pauline Jaricot est bien éloignée du socialisme utopique d'un Cabet ou d'autres utopistes qui rêvent d'un communisme chrétien. Ce n'est pas une révolutionnaire et ses projets ouvriers n'ont qu'un objectif : étendre l'influence de l'Église sur cette classe ouvrière qui lui échappe et qui devient perméable au discours socialiste - influence qu'elle dénonce dans plusieurs de ses circulaires. Pour contrer cette influence, elle préconise même de former des "ouvriers apôtres» qui iraient dans les usines combattre la propagande socialiste. Cette lettre reprend les diverses analyses développées sur la condition ouvrière, depuis 1832 , dans ses circulaires aux associés du Rosaire. Bien qu'elle rejoigne souvent les positions de l'Église, la vision développée par Pauline Jaricot, très critique sur l'utilisation par les possédants de leurs richesses, est perçue par la bourgeoisie lyonnaise comme une attaque. Cette critique concerne aussi implicitement le clergé séculier, qui, à Lyon, selon elle, ne remplit pas sa fonction de protection des plus pauvres. Pour Pauline Jaricot, il y aurait même une certaine complicité, au moins passive, de la part de ces clercs laissant la classe ouvrière dans une position de "dépendance et d'abandon" alors que la fonction sociale de l'Église est de protéger les plus démunis. Cette passivité permet, dit-elle, au "Capitalisme» d'imposer ses règles. La critique de la socié- 
té industrielle en formation conduit Pauline Jaricot à valoriser les pauvres qui seraient plus réceptifs au message évangélique d'où le thème du "Jésus ouvrier", fréquent en 1848 , et une relecture de l'évangile centrée sur le partage équitable des richesses : «l'or et l'argent doivent donc avoir une bonne destination». Ses critiques ne sont guère éloignées de celles que Lamennais formulait en 1834 dans "Paroles d'un croyant»:

La miséricorde du Christ est sans exception. Il est venu en ce monde pour sauver non quelques hommes mais tous les hommes; il a eu pour chacun d'eux une goutte de sang. Mais les petits les faibles, les humbles, les pauvres, tous ceux qui souffraient, il les aimait d'un amour de prédilection. Son cœur battait sur le cœur du peuple, et le cœur du peuple battait sur son cœeur.

Pauline Jaricot développe sa conception d'une Église au service des pauvres dans de nombreux écrits - lettres, circulaires aux associés du Rosaire - après l'échec de l'CEuvre des ouvriers. Pour contrer son influence, des membres du clergé vont alors la présenter comme soumise aux influences des méthodistes (protestants). Alors que l'Église-institution ne propose rien, selon elle, pour améliorer les conditions de vie des ouvriers, elle propose une réforme qui permette de "rendre à l'ouvrier sa dignité d'homme». L'échec de la fonderie de Rustrel qu'elle croit passager - ne remet pas en cause ses théories sociales, bien au contraire. En quelques phrases, Pauline Jaricot trace pour l'Église un véritable programme politique qui s'articule autour de trois axes. D'abord permettre aux hommes de retrouver leur dignité avant de parler d'évangélisation : «soulagez, aimez premièrement et moralisez ensuiten ; puis faire en sorte que l'ouvrier puisse vivre dignement de son travail : «jouir des avantages d'un travail réglé avec prudence et rétribué selon la justice" ; enfin instruire les enfants du milieu populaire - garçons et filles - afin de "développer l'intelligence, ne laisser improductif aucun don [...]. Aussi toutes les carrières, leur seront ouvertes". Un projet qui est finalement assez proche de l'ordre économique dessiné par les premiers socialistes et qui, par bien des aspects s'apparente à la cité idéale d'Owen. Mais le monde qu'elle appelle de ses vœux, où l'évangélisation reposerait avant tout sur des ouvriers prédicateurs, est difficilement pensable pour l'Église.

5 Félicité de Lamennais, Paroles d'un croyant, édition originale, Paris, E. Renduel, 1834. Texte reproduit dans Socialisme et Utopies de Babeufa aa Jaurès, La Documentation photographique, bimestriel $n^{\circ} 8016$, août 2000, La Documentation française. 
Le drame de Pauline Jaricot transparaît en filigranne dans cette lettre. En tant que femme, laïque de surcroît, elle s'immisce dans le débat sur la condition ouvrière qui traverse alors l'Église. Ses analyses sur les causes de la paupérisation et les remèdes qu'elle propose n'étant pas relayés par une congrégation ou même par des prêtres, demeurent confidentiels. Les autorités catholiques de 1850 retiennent d'abord son échec de Rustrel et ses divers écrits ne sont ni repris ni publiés après sa mort en 1861 .

Pauline Jaricot ne deviendra une figure importante, pour l'Église catholique, qu'après un temps d'oubli. Ainsi, en 1881, alors que l'Église, soucieuse de reconquérir la classe ouvrière, cherche à mettre en évidence une préoccupation déjà ancienne face à la paupérisation du monde ouvrier, Pauline Jaricot peut être mise en avant sans crainte, pour plusieurs raisons. Elle a toujours placé sa démarche dans une stratégie d'évangélisation; elle présente les atouts requis pour devenir une sainte : inconnue en dehors d'un cercle restreint de catholiques, elle est passée du statut de riche bourgeoise à celui d'indigente, par amour des ouvriers ; enfin, elle aurait, avant les socialistes de la fin du XIX" siècle, dénoncé les méfaits du capitalisme sauvage et proposé des solutions. L'utilisation de son image est d'autant plus aisée que personne ne connaît ses écrits. Ce qui évite de faire le procès d'une partie du clergé qui l'a menée à la ruine.

\section{Lettre du 3 Décembre 1848 adressée à Julia Maurin}

Je dirai mes vues et mes désirs à l'égard de toutes les classes de la société, pour fortifier la grande famille des chrétiens dans les temps actuels et surtout dans ceux qui toucheront à la fin des siècles. [...]

Ce qui frappe le plus tout homme animé d'un esprit de justice et d'humanité dans l'examen de la situation de la classe ouvrière, c'est l'état de dépendance et d'abandon dans lequel la société livre les ouvriers aux chefs et aux entrepreneurs des manufactures ; c'est la faculté illimitée de laisser à des capitalistes spéculateurs de réunir autour d'eux des populations entières pour en employer les bras suivant leur intérêt, pour en disposer en quelque sorte à discrétion, sans qu'aucune garantie d'existence, d'avenir d'amélioration morale et physique soit donné de leur part, ni à la société qui doit les protéger.

Plus je fréquentais les pauvres plus il m'était donné d'approfondir les maux qui dévorent la société. Il m'a été permis de voir en Dieu et avec une douleur profonde, que les grands ravages causés par son ennemi et celui des âmes proviennent de l'abus de l'or. L'or et l'argent, dont le dragon de l'abîme fait sa litière, ne 
s'entassent à cette heure que pour répandre au loin la peste des mauvaises doctrines et la corruption des mours. [...]

À la vue de tous ces éléments de perturbation combinés par Satan, et de ces ruses pour tarir les sources auxquelles la charité s'est toujours abreuvée, mon âme s'est émue d'une profonde indignation contre l'enfer. Alors, m'humiliant aux pieds de notre bon Maître, j’ai osé lui dire : O Jésus! je sais que vous n’avez racheté le monde ni avec l'or ni avec l'argent, mais avec votre sang et vos larmes. En vous faisant homme, vous avez choisi la pauvreté et le travail, afin de guérir toutes convoitises de la nature déchue. Malgré cela, permettez-moi, Seigneur si miséricordieux, de vous dire toute ma pensée. Après avoir formé et coordonné toutes choses, vous avez dit que tout était bon. L'or et l'argent doivent donc, dans les desseins de votre Providence, avoir une bonne destination, puisque, comme l'ensemble des êtres et des choses, ils ne sont sortis de vos mains que pour servir votre gloire. Pourtant, cher Maître, voyez à quelles fins on en use! Pourquoi ces créatures, innocentes par elles-mêmes, sont-elles ainsi violentées dans leurs biens ? faites cesser une si grande perturbation et pour cela, rendez ces matières à leur destination primitive. Si la compassion émeut ma pauvre âme en faveur de la partie laborieuse de votre peuple, ne vous en fâchez pas. [...]

À mesure que l'industrie s'exerce à remplacer les bras de l'homme par des machines, l'ouvrier vertueux qui savait et pouvait autrefois faire des économies pour l'avenir et appeler, pas ses aumônes, les bénédictions d'en-Haut sur son travail, ne connaît aujourd'hui que la gêne : le prix des journées ne peut plus suffire aux besoins multipliés de sa famille. Beaucoup sont frustrés, en un seul jour, de petites épargnes de toute leur vie, par des faillites frauduleuses; ce qui donne à d'autres la pensée de garder leurs faibles ressources sans les faire valoir, pour ne pas s'exposer à les perdre ainsi. Pendant ce temps les riches contentent leurs besoins de jouissances et la séduction de l'or étend son empire et le nombre de ses victimes.

Je cherche devant Dieu le moyen de remédier au découragement, à l'immoralité et à l'exaspération qui se manifestent de plus en plus dans les masses. Il me semble aujourd'hui avoir acquis la certitude qu'il faudrait d'abord rendre à l'ouvrier sa dignité d'homme, en l'arrachant à l'esclavage d'un travail sans relâche ; sa dignité de père en lui faisant goûter les douceurs et les charmes de la famille; sa dignité de chrétien en lui procurant, avec les joies du foyer domestique, les consolations et les espérances de la Religion. En un mot, je voudrais qu'on rendit l'époux à l'épouse, le père à l'enfant et Dieu à l'homme [...]

On obtiendrait doucement ce retour salutaire, si l'on s'adressait d'abord au 
cœur de l'ouvrier, pour arriver ensuite à son âme. Quand le cœur est gagné par la reconnaissance, il s'ouvre de lui-même à la lumière qu'on veut y faire pénétrer. C'est en vain qu'on essaie de moraliser le peuple en s'adressant à son esprit [...] Si vous voulez moissonner cent pour un, soulager, aimez premièrement et moralisez ensuite.

J'entrevis le moyen de former un établissement où des ouvriers vertueux pourraient en famille jouir des avantages d'un travail réglé avec prudence et rétribué selon la justice, expérimentant que, dans tout état créé par Dieu, il a des moyens faciles de salut. Là, me disais-je, le père de famille verra ses enfants s'élever au foyer paternel, sans être privés pour cela de l'éducation convenable à leur avenir $[\ldots]$. Cette éducation première doit avoir un cachet de vigueur et de désintéressement. Donner à l'enfant, non la satisfaction de tous ses désirs [...] d'où naissent l'égoïsme et la mollesse, mais l'habitude du sacrifice et l'amour du devoir. Agir avec une grande douceur et une exquise délicatesse [...] il faut incliner la volonté et le cœur de l'enfant de manière à lui rendre facile et doux ce qui lui serait naturellement difficile et insupportable [...]. En développant en eux les facultés de l'âme, on développera avec le même soin celles de l'intelligence, afin de ne laisser improductif aucun des dons que Dieu leur aura fait. Aussi toutes les carrières, même celles des arts leur seront ouvertes. [...]

Après s'être fortifiés dans le combat contre eux-mêmes, ils iront plus tard se mesurer contre les mêmes passions, pour en délivrer leurs frères, par la vertu toute-puissante de Jésus-Ouvrier. Notre-Dame des Anges sera une pépinière d'ouvriers vertueux qui iront propager le bon esprit dans tous les ateliers où l'on sera bien aise de les recevoir pour cette fin, avec faculté de revenir dans leur établissement au sein de la famille. J'ai vu les effets de la puissance de ce zèle dans les âmes simples et droites avec lesquelles j'ai été, dès ma jeunesse, liée d'amitié et de bonnes ouvres. [...] Après tout, pourquoi n'essaierait-on pas de rallumer, par cet apostolat de charité, la religion dans la masse ? [...] 